\title{
La autoevaluación es un método poco confiable para establecer el desarrollo puberal en escolares
}

\author{
CRISTIAN MARCHANT F. ${ }^{1}$, RODRIGO BANCALARI D. ${ }^{1}$, CARLOS DÍAZ S. ${ }^{2}$, \\ JUANITA ZAMORANO R. ${ }^{3}$, VERÓNICA CERDA F. ${ }^{3}$, MANUEL FERNÁNDEZ V. ${ }^{3}$, \\ FLAVIA GARBIN A. ${ }^{3}$, PATRICIA MUÑOZ C. DEL V. ${ }^{3}$, GABRIEL CAVADA C. ${ }^{4}$, HERNÁN GARCÍA B. ${ }^{1}$ \\ 1. Departamento de Pediatría, Unidad de Endocrinología, Escuela de Medicina, Pontificia Universidad Católica de Chile. \\ 2. Programa de Doctorado, Escuela de Salud Pública, Facultad de Medicina, Universidad de Chile. \\ 3. Departamento de Pediatría, Universidad de los Andes. \\ 4. Departamento de Salud Pública, Universidad de los Andes.
}

\begin{abstract}
Self evaluation is not reliable for assessing puberal development in school-aged children

Introduction: Puberal development assessment (PDA) is performed according Tanner's method (TM). Objective: In order establish the coincidence between PDA determined by physicians and the self-evaluation by school-aged children. Material and Methods: 2980 school children from Santiago, Chile, were assessed by means of TM, the development of the mammary gland (MD), male genitalia (MG) and pubic hair (PH) were assessed. PDA was simultaneously performed by physicians and by the school children. Results: Concordance between physicians and self assessment showed a kappa coefficient (KC) of $0.55,0.45$, and 0.51 in PH, MD and MG respectively, (acceptable $\mathrm{KC}>0.61$ ). Self-evaluation of PDA decreased as the age of children increased, with OR of 0.76 (95\% IC $0.74-0.79)$; 0.87 (95\% IC $0.83-0.91)$ and 0.92 (95\% IC $0.88-0.96)$ for PH, $\mathrm{MD}$ and MG respectively. An inverse relationship between nutritional status (NS) and PDA was observed only in PH, obese school children underscored their PH (OR 0.6; 95\% IC 0.5 - 0.7). Multivariate analysis for gender and NS showed that only females overestimate their PH, OR of 1.15 (95\% IC 1-1.32). Conclusions: PDA through self-assessment yields only moderate correlation coefficients, thus it is not reliable for making relevant clinical decisions.
\end{abstract}

(Key words: Self assessment, mammary development, genitalia, pubic hair).

Rev Chil Pediatr 2012; 83 (4): 345-351

\section{RESUMEN}

Introducción: La determinación del desarrollo puberal (DDP) se evalúa según el método de Tanner (MT). Objetivo: Determinar la concordancia de la DDP entre médicos con la autoevaluación en escolares. Pacientes y Métodos: Se examinaron 2980 escolares de Santiago de Chile. Se evalúo desarrollo mamario (DM), genitales masculinos (GM) y vello púbico (VP) mediante el MT. La DDP fue evaluada simultáneamente por un médico y por los escolares. Resultados: La concordancia entre médicos y la autoevaluación mostró un coeficiente kappa (CK) de 0,55, 0,45, 0,51 en VP, DM y GM respectivamente, (CK aceptable $>0,61)$. La autoevaluación del DDP disminuyo a medida que aumentaba la edad, con OR respectivos de 0,76 (95\% IC

Proyecto MED 2006, financiado por Fondo de Ayuda a la Investigación (FAI) Universidad de los Andes.

Recibido el 18 de mayo de 2011, devuelto para corregir el 04 de julio 2011, segunda versión el 06 de febrero de 2012, aceptado para publicación el 05 de abril 2012.

Correspondencia a:

Dr. Hernán García B.

E-mail:hgarciab@med.puc.cl 
0,74-0,79); 0,87 (95\% IC 0,83-0,91) y 0,92 (95\% IC 0,88 -0,96) para VP, DM y GM. Se observo una relación inversa entre estado nutricional (EN) y DDP sólo en VP, escolares obesos subestimaban su VP (OR 0,6; 95\% IC 0,5-0,7). El análisis multivariado de género y EN mostró que sólo las mujeres sobreestimaban su VP, OR de 1,15 (95\% IC 1-1,32). Conclusiones: La DDP mediante autoevaluación obtiene coeficientes de correlación sólo moderados que no permiten confiar en este para establecer decisiones clínicas relevantes.

(Palabras clave: Autoevaluación, desarrollo mamario, genitales, vello púbico, Tanner).

Rev Chil Pediatr 2012; 83 (4): 345-351

\section{Introducción}

La determinación precisa del desarrollo puberal es un elemento importante del examen físico en niños y adolescentes, siendo parte fundamental de toda evaluación del estado de salud en este grupo etario. En el año 1962 Tanner creó un método que compara desarrollo de los caracteres sexuales secundarios del paciente evaluado con fotografías estandarizadas que van entre el grado 1 (ausencia de desarrollo) hasta el 5 (desarrollo completo) ${ }^{1,2}$, Este método considera sólo la inspección, y no establece necesidad de palpación; pero obviamente mejora si se agrega palpación de tejido mamario y medición de volumen testicular utilizando orquidómetro. El método de Tanner tiene gran utilidad en la práctica clínica, pero tiene como desventaja el requerir observar directamente las mamas en las niñas y los genitales en los varones, lo que es incómodo para los pacientes especialmente si el observador es del sexo opuesto, y difícil de aplicar en evaluaciones grupales (por ejemplo colegios, instituciones de menores, etc.).

La autoevaluación del desarrollo puberal realizada por el propio paciente en base a las mismas fotografías ha sido planteada como una posible alternativa en estos casos. Sin embargo, los estudios disponibles son escasos y han sido realizados con muestras pequeñas y con resultados dispares ${ }^{3-7}$, En la actualidad no existe un consenso para definir que método es el más preciso para realizar la autoevaluación, por lo que esta pregunta no se encuentra contestada aun satisfactoriamente.

El objetivo del presente estudio es evaluar la concordancia existente entre la evaluación del desarrollo puberal mediante método de Tanner, efectuada por médico pediatra con la autoevaluación realizada por escolares sanos, así como estudiar la influencia del género, edad y estado nutritivo en la autodeterminación.

\section{Pacientes y Método}

\section{Diseño y muestra}

Estudio de base poblacional y de corte transversal para evaluar estado nutritivo y desarrollo puberal en escolares chilenos.

Se seleccionó una muestra de 3010 escolares de 6 a 15 años pertenecientes a 10 comunas de Santiago de estrato socioeconómico mediobajo cuyo universo fueron todos los escolares pertenecientes a los colegios con educación básica de la Sociedad de Instrucción Primaria (SIP) que cursaban en el año 2006 de primero a octavo básico. Esta institución cuenta con quince colegios de educación básica, distribuidos aleatoriamente en 10 comunas de la ciudad de Santiago y cuentan con una población escolar de educación básica de aproximadamente 15000 alumnos.

Con el objetivo de obtener una muestra representativa de los 15000 alumnos que componen la institución estudiada (SIP), la selección de los sujetos se realizó mediante un muestreo aleatorio, multietápico y por conglomerados. Los conglomerados estuvieron constituidos por los colegios. Se seleccionaron mediante aleatorización simple 10 de los 15 colegios que cuentan con educación básica, los cuales quedaron distribuidos en 9 comunas de la capital; 4 colegios en el área sur, 3 colegios en el área central y 3 colegios en el área norte. En los establecimientos elegidos se realizó un muestreo aleatorio estratificado por sexo y curso, invitándose a participar a 336 alumnos por cada colegio. Para la generación de números al azar se utilizó el servicio denominado "True Random Number Service" mantenido por el Colegio de Estadísticos y Ciencias Computa- 
cionales del "Trinity College" en Dublin, Irlanda y disponible para su uso en Internet.

Los criterios de inclusión fueron estar inscritos en los cursos de primero a octavo básico de los colegios de la SIP y que sus padres o tutores hayan aceptado que sus hijos participen de este estudio. Quedaron excluidos aquellos alumnos que no fueran autorizados por sus padres o tutores, así como también los sujetos que no entregaron su consentimiento. El trabajo de campo se llevó a cabo entre marzo y diciembre del año 2006. Treinta alumnos fueron excluidos por no presentar consentimiento, en este trabajo no se utilizó asentimiento. Veintiocho alumnos rechazaron participar a pesar de tener el consentimiento. Por lo tanto, finalmente se excluyeron 58 alumnos.

Para la realización de este estudio se contó con 8 pediatras y 16 internos de medicina los cuales fueron capacitados en la evaluación antropométrica (correspondientes a alumnos de sexto año de la carrera de medicina de la Universidad de los Andes, que se encontraban en su rotación por pediatría), en módulos de examen simultáneos, durante la jornada escolar. La evaluación de desarrollo puberal se realizó siempre por un médico pediatra.

\section{Evaluación antropométrica}

Se determinó la talla en posición erecta empleando un estadiómetro de pared fijo marca SECA ${ }^{\circledR}$, se codificó en centímetros más un decimal, realizándose tres mediciones. El resultado final se consignó como el promedio de las tres mediciones. Se pesó a los niños descalzos, con ropa interior, se realizaron tres mediciones con pesa marca SECA modelo 700, previamente calibrada mediante la utilización de pesos fijos conocidos, determinándose el peso en kilos con un decimal. El resultado final se consignó como el promedio de las tres mediciones.

\section{Estado nutricional}

El bajo peso, el sobrepeso y la obesidad se definieron mediante el IMC (peso/talla ${ }^{2}$ ). Se consideró bajo peso un IMC bajo el percentil 10 , sobrepeso un IMC entre el percentil 85 y 95, y obeso un IMC por encima del percentil 95 , los valores obtenidos de IMC se compa- raron tablas estándares de la NCHS según la edad y el sexo del alumno ${ }^{8}$.

\section{Evaluación de desarrollo puberal}

Para la evaluación del desarrollo puberal, cada alumno fue evaluado por un médico pediatra de su mismo sexo, examinándose mediante inspección el desarrollo mamario en niñas, genitales en varones y vello pubiano en ambos sexos. No se midió volumen testicular por no haber autorización expresa en el consentimiento informado. Se utilizaron las fotografías de los cinco estadios de Tanner ${ }^{1,2}$ para clasificar al escolar según su desarrollo sexual alcanzado. Para respetar la privacidad y promover la comodidad de cada niño, se examinó en ausencia de otros niños presentes, en una sala especialmente habilitada.

El grado de desarrollo puberal alcanzado, se evaluó clasificando a los escolares en alguno de los 5 estadios de Tanner descritos para ambos sexos.

Paralelamente a todos los participantes se les solicitó autoevaluar su desarrollo puberal mostrándoles las mismas fotografías de los estadios de Tanner utilizadas por los examinadores, pidiéndoles a continuación que indicaran en el estadio en que ellos o ellas creían encontrarse.

\section{Análisis estadístico}

Los datos obtenidos fueron evaluados mediante análisis kappa para determinar la concordancia de la autoevaluación versus los médicos pediatras. El coeficiente kappa ( $\kappa)$ toma valores entre $-1 \mathrm{y}+1$; mientras más cercano a +1 , mayor es el grado de concordancia interobservador. Por el contrario, un valor de $\kappa=$ 0 refleja que la concordancia observada es precisamente la que se espera a causa exclusivamente del azar. La interpretación del coeficiente kappa se realiza correlacionando su valor con una escala cualitativa que incluye seis niveles de fuerza de concordancia ("pobre", "leve", "aceptable", "moderada", "considerable" y "casi perfecta"), simplificando la comprensión del mismo ${ }^{9}$. Para variantes continuas (índice de masa corporal) se realizó análisis con desviaciones estándar.

Se evaluó la concordancia general (outco- 
me primario) y según sexo, estado nutricional (IMC) y grado de desarrollo puberal (prepúber $v s$ desarrollo iniciado, definido como grado de Tanner $1 \mathrm{y} \geq 2$, respectivamente).

\section{Análisis de factores que pudieran influir en la autoevaluación del desarrollo puberal}

Para analizar el efecto que pudieran tener las distintas variables en la autoevaluación se midió mediante ajuste por regresión logística la influencia de la edad, estado nutricional (definido para este análisis como eutrofia, sobrepeso y obesidad) y el género como variables individuales. Éste último se analizó solamente en referencia al vello púbico ya que era la única variable que fue medida en ambos sexos. Asimismo se evalúo mediante el mismo método la influencia del género y estado nutricional en la evaluación del vello púbico.

Para la confección de la base de datos el análisis estadístico se utilizó el programa STATA 11.0 (Statacorp, College Station, Texas, estados Unidos).

\section{Aspectos éticos}

El estudio fue aprobado por los colegios participantes y el consentimiento informado fue otorgado por los padres o tutores.

\section{Resultados}

Características de la población estudiada

Se reclutaron 2980 escolares, 1433 muje- res y 1547 hombres, con una edad promedio de 10,3 $\pm 2,3$ años. Los z-score de talla e IMC fueron de $-0,06 \pm 0,9$ y de $0,79 \pm 1$ respectivamente (tabla 1).

\section{Desarrollo puberal}

De los 2980 pacientes reclutados, 2952 $(99,1 \%)$ aceptaron ser evaluados para determinar su desarrollo puberal. Se calculo las diferencias entre la apreciación de desarrollo de vello pubiano según género (único parámetro evaluado en ambos sexos).

Los resultados comparados de la autoevaluación con la realizada por los médicos pediatras, en vello púbico en ambos sexos, mamas y genitales masculinos se observan en las figuras 1,2 y 3 .

\section{Análisis de concordancia}

El cálculo del coeficiente kappa para evaluar la concordancia entre la evaluación por personal de salud y la autoevaluación mostró los siguientes resultados:

a) Vello púbico: se obtuvo un coeficiente kappa de 0,55 general. Al desglosar por género, el coeficiente kappa no fue diferente según sexo, siendo de 0,57 en hombres y 0,58 en mujeres.

b) Desarrollo mamario: el coeficiente kappa resultante fue de 0,45.

c) Genitales masculinos: el análisis del coeficiente kappa arrojó un resultado de 0,51. (tabla 2).

Tabla 1. Características generales de la población de estudio

\begin{tabular}{|c|c|c|c|c|c|c|c|c|c|c|c|c|c|c|c|}
\hline \multirow{2}{*}{$\begin{array}{l}\text { Género } \\
\text { Estado } \\
\text { nutricional }\end{array}$} & \multicolumn{5}{|c|}{ Femenino } & \multicolumn{5}{|c|}{ Masculino } & \multicolumn{5}{|c|}{ Total } \\
\hline & $\begin{array}{l}\text { Bajo } \\
\text { peso }\end{array}$ & $\begin{array}{c}\text { Eutró- } \\
\text { fico }\end{array}$ & $\begin{array}{c}\text { Sobre } \\
\text { peso }\end{array}$ & Obeso & Total & $\begin{array}{l}\text { Bajo } \\
\text { peso }\end{array}$ & $\begin{array}{l}\text { Eutró- } \\
\text { fico }\end{array}$ & $\begin{array}{c}\text { Sobre } \\
\text { peso }\end{array}$ & Obeso & Total & $\begin{array}{l}\text { Bajo } \\
\text { peso }\end{array}$ & $\begin{array}{l}\text { Eutró- } \\
\text { fico }\end{array}$ & $\begin{array}{l}\text { Sobre } \\
\text { peso }\end{array}$ & Obeso & Total \\
\hline$n,(\%)$ & $\begin{array}{c}25 \\
(1,74)\end{array}$ & $\begin{array}{c}799 \\
(55,7)\end{array}$ & $\begin{array}{c}339 \\
(23,6)\end{array}$ & $\begin{array}{c}270 \\
(18,8)\end{array}$ & $\begin{array}{l}1433 \\
(100)\end{array}$ & $\begin{array}{c}20 \\
(1,27)\end{array}$ & $\begin{array}{c}794 \\
(50,5)\end{array}$ & $\begin{array}{c}381 \\
(24,3)\end{array}$ & $\begin{array}{c}352 \\
(22,4)\end{array}$ & $\begin{array}{l}1547 \\
(100)\end{array}$ & $\begin{array}{c}45 \\
(1,51)\end{array}$ & $\begin{array}{l}1593 \\
(53,5)\end{array}$ & $\begin{array}{c}720 \\
(24,2)\end{array}$ & $\begin{array}{c}622 \\
(20,9)\end{array}$ & $\begin{array}{l}2980 \\
(100)\end{array}$ \\
\hline $\begin{array}{l}\text { Edad, } \\
\text { años** }\end{array}$ & $\begin{array}{l}10,6 \\
\pm \\
2,4\end{array}$ & $\begin{array}{c}10,5 \\
\pm \\
2,4\end{array}$ & $\begin{array}{c}10,4 \\
\pm \\
2,3\end{array}$ & $\begin{array}{c}9,9 \\
\pm \\
2,3\end{array}$ & $\begin{array}{c}10,4 \\
\pm \\
2,4\end{array}$ & $\begin{array}{c}10,6 \\
\pm \\
2\end{array}$ & $\begin{array}{c}10,6 \\
\pm \\
2,3\end{array}$ & $\begin{array}{c}10,4 \\
\pm \\
2,4\end{array}$ & $\begin{array}{l}9,7 \\
\pm \\
2,2\end{array}$ & $\begin{array}{c}10,3 \\
\pm \\
2,3\end{array}$ & $\begin{array}{c}10,6 \\
\pm \\
2,2\end{array}$ & $\begin{array}{c}10,6 \\
\pm \\
2,3\end{array}$ & $\begin{array}{c}10,4 \\
\pm \\
2,4\end{array}$ & $\begin{array}{l}9,8 \\
\pm \\
2,4\end{array}$ & $\begin{array}{c}10,3 \\
\pm \\
2,3\end{array}$ \\
\hline $\begin{array}{l}\text { Talla, puntaje } \\
z^{* *}\end{array}$ & $\begin{array}{c}-0,06 \\
\pm \\
1\end{array}$ & $\begin{array}{c}-0,27 \\
\pm \\
0,9\end{array}$ & $\begin{array}{c}0,06 \\
\pm \\
0,9\end{array}$ & $\begin{array}{c}0,27 \\
\pm \\
0,9\end{array}$ & $\begin{array}{c}-0,09 \\
\pm \\
0,9\end{array}$ & $\begin{array}{c}-0,48 \\
\pm \\
1\end{array}$ & $\begin{array}{c}-0,26 \\
\pm \\
0,9\end{array}$ & $\begin{array}{c}0,10 \\
\pm \\
1\end{array}$ & $\begin{array}{c}0,34 \\
\pm \\
0,9\end{array}$ & $\begin{array}{c}-0,03 \\
\pm \\
1\end{array}$ & $\begin{array}{c}-0,24 \\
\pm \\
1\end{array}$ & $\begin{array}{c}-0,27 \\
\pm \\
0,9\end{array}$ & $\begin{array}{c}0,08 \\
\pm \\
0,9\end{array}$ & $\begin{array}{c}0,31 \\
\pm \\
0,9\end{array}$ & $\begin{array}{c}-0,06 \\
\pm \\
0,9\end{array}$ \\
\hline $\begin{array}{l}\text { IMC, puntaje } \\
z^{* *}\end{array}$ & $\begin{array}{c}-2,33 \\
\pm \\
0,8\end{array}$ & $\begin{array}{c}0,20 \\
\pm \\
0,6\end{array}$ & $\begin{array}{l}1,32 \\
\pm \\
0,2\end{array}$ & $\begin{array}{l}1,95 \\
\pm \\
0,2\end{array}$ & $\begin{array}{l}0,75^{*} \\
\pm \\
1\end{array}$ & $\begin{array}{c}-2,16 \\
\pm \\
0,6\end{array}$ & $\begin{array}{c}0,15 \\
\pm \\
0,6\end{array}$ & $\begin{array}{c}1,33 \\
\pm \\
0,2\end{array}$ & $\begin{array}{l}1,97 \\
\pm \\
0,3\end{array}$ & $\begin{array}{c}0,82 \\
\pm \\
1\end{array}$ & $\begin{array}{c}-2,26 \\
\pm \\
0,7\end{array}$ & $\begin{array}{c}0,18 \\
\pm \\
0,6\end{array}$ & $\begin{array}{c}1,32 \\
\pm \\
0,2\end{array}$ & $\begin{array}{c}1,96 \\
\pm \\
0,3\end{array}$ & $\begin{array}{c}0,79 \\
\pm \\
1\end{array}$ \\
\hline
\end{tabular}

* = Valor $p$ entre grupo femenino y masculino; $=p<0,05 ; * *=$ Promedio y desviación estandar. 


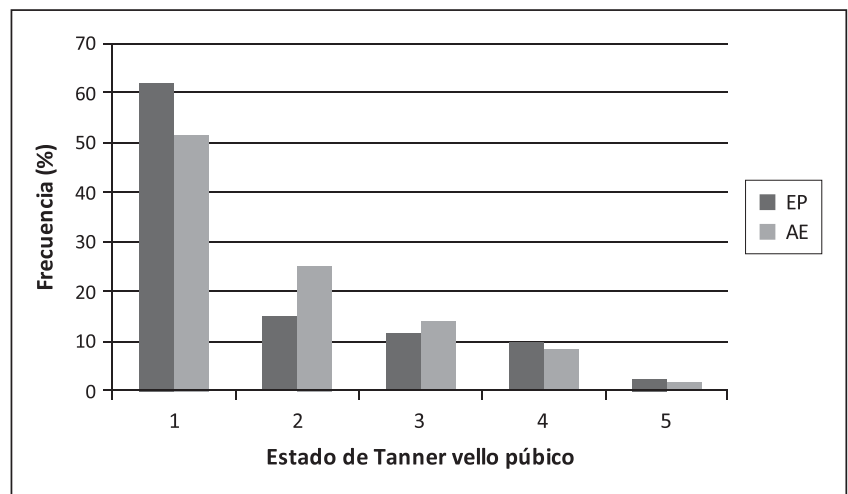

Figura 1. Evaluación del desarrollo de vello púbico. EP: Evaluación por pediatra, AE: Autoevaluación.

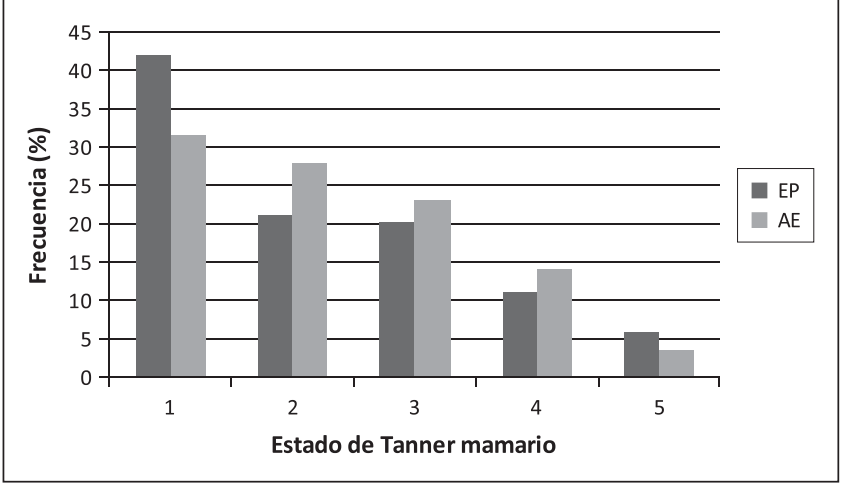

Figura 2. Evaluación del desarrollo mamario. EP: Evaluación por pediatra, AE: Autoevaluación.

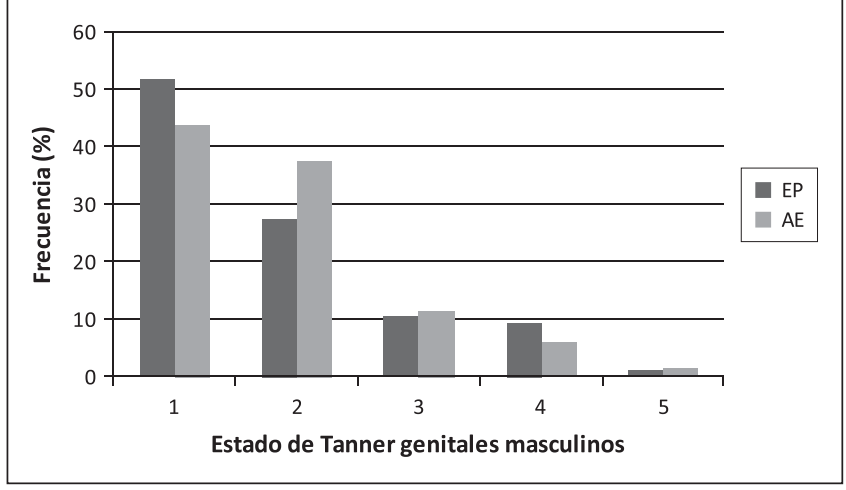

Figura 3. Evaluación del desarrollo de genitales masculinos. EP: Evaluación por pediatra, AE: Autoevaluación.

\section{Evaluación de influencia de variables estudiadas en la autoevaluación (análisis univariable)}

a) Edad y género: la edad fue un factor estadísticamente significativo, para autoevaluarse en una etapa de Tanner inferior a la evaluada por pediatra a medida que se tenía más edad. Esto fue consistente en las evaluaciones de vello púbico, mamas y genitales masculinos con OR respectivos de $0,76(95 \%$ IC $0,74-0,79) ; 0,87$ (95\% IC 0,83-0,91) y 0,92 (95\% IC 0,88-0,96).

Respecto al género, su influencia sólo se puede evaluar en vello púbico presente en ambos sexos, donde la autoevaluación fue diferente entre hombres y mujeres existiendo una diferencia significativa, la concordancia de los alumnos hombres con respecto al evaluador es de $74,1 \%$ y de las mujeres de un 69,9\% $(\mathrm{p}=0,011)$.

b) Estado nutricional: al medir el efecto del estado nutricional en la autoevaluación, la obesidad tuvo un efecto significativo en la evaluación del vello púbico, observándose una relación inversa, donde los escolares obesos tendían a subestimar su Tanner de vello púbico (OR 0,6; 95\% IC 0,5$0,7)$. En mamas y genitales masculinos se observó una tendencia similar, pero sin alcanzar resultados estadísticamente significativos.

c) Estado nutricional y género (análisis multivariable): el análisis del efecto combinado del género y el estado nutricional en la autoevaluación del vello púbico mostró que las mujeres obesas tendían a sobreestimar su Tanner de vello púbico, lo cual fue estadísticamente significativo con un OR de 1,15 (95\% IC 1-1,32) y no se observó en los varones.

\section{Discusión}

Los resultados obtenidos en este estudio con una muestra grande de escolares chilenos demuestran que la autoevaluación del desarrollo puberal utilizando los grados de la escala de Tanner no es del todo confiable, obteniéndose coeficientes de correlación kappa sólo moderados (como consenso se consideran coeficientes buenos 
MARCHANT C. y cols.

Tabla 2. Concordancia entre evaluación por pediatra y autoevaluación (Análisis de concordancia Kappa)

\begin{tabular}{|lccccc|}
\hline Variable & $\begin{array}{c}\text { Concordancia } \\
\text { (\%) }\end{array}$ & Kappa & $\begin{array}{c}\text { Error estándar del Kappa } \\
\text { encontrado }\end{array}$ & z & Prob > z \\
\hline Genitales & 68,3 & 0,51 & 0,01 & 30,7 & 0,00 \\
Mamas & 58,9 & 0,45 & 0,01 & 30,7 & 0,00 \\
Vello púbico & & & & 49,1 & 0,00 \\
Total & 72 & 0,55 & 0,01 & 35,1 & 0,00 \\
Hombres & 74,2 & 0,57 & 0,01 & 35 & 0,00 \\
Mujeres & 72,9 & 0,58 & 0,01 & & \\
\hline
\end{tabular}

los mayores a $\left.0,61^{9}\right)$. Nosotros obtuvimos un coeficiente de correlación en vello púbico total de 0,58 y al desglosar por sexo obtuvimos en vello púbico hombres 0,57 y en vello púbico mujeres 0,58 . Al evaluar genitales masculinos 0,51 y por último desarrollo mamario de 0,45.

Con respecto a las variables que tuvieron influencia sobre la autoevaluación, se observa influencia de la edad, género y estado nutricional. Respecto de la edad, a mayor edad se tiende a subestimar el desarrollo puberal, lo que podría deberse a que en niños mayores el desarrollo puberal efectivamente es más avanzado, por lo que si hay error la tendencia natural es hacia la subestimación de éste. Se podría especular en forma adicional que los niños mayores podrían subestimar su desarrollo puberal por un factor de pudor o vergüenza, sin embargo, esta variable no se evaluó con alguna escala de medición, cuestionario o evaluación psicológica validada, por lo que no es posible confirmar esta hipótesis con los datos disponibles.

Por otra parte, nuestro trabajo presenta un sesgo, para el análisis de concordancia, ya que los escolares que estaban en etapas de desarrollo más avanzadas (Tanner $\geq 3$ ) eran menos que los niños en etapa prepuberal o en estado inicial (Tanner 1 y 2), lo que podría influir en los resultados obtenidos ya que con un número menor de participantes, las tendencias obtenidas se hacen más evidentes que con más individuos, y el coeficiente kappa, que es un análisis de probabilidad de concordancia entre observadores, hace más notoria esta discordancia.

Con respecto a la variable estado nutricional, llama la atención la tendencia observada en los escolares obesos a subestimar su desarrollo de vello púbico, mamas y genitales masculinos. Si bien esto era esperable en varones obesos debido al aumento de su panículo adiposo peri genital, que podría ocultar su pene, lo que explicaría que subestimaran su desarrollo genital, llamó la atención que las niñas obesas también subestimaran su desarrollo mamario, ya que se esperaba que iban a tender a sobreestimarlo por la presencia de lipomastia. Esto podría atribuirse a que las niñas más desarrolladas que sus pares podrían desear poseer menor desarrollo mamario, pero como ya se mencionó no realizamos alguna evaluación en esa esfera, por lo que no es posible corroborar esta hipótesis.

No existe una explicación clara del porque las niñas obesas sobreestiman su desarrollo de vello púbico y sólo corrobora la pobre concordancia observada en nuestro estudio entre autoevaluación de Tanner vs evaluación por pediatra.

El presente estudio tiene como principal fortaleza el gran número de individuos evaluados, lo cual lo diferencia de estudios previamente publicados $^{3-7}$. Por otro lado, la interpretación de los resultados obtenidos es más difícil al no haberse realizado otras mediciones como volumen testicular, palpación y medición de tejido mamario, así como cuestionarios o evaluaciones psicológicas, que apoyen las explicaciones postuladas. Esto fue realizado y reportado en un grupo de 366 niños y adolescentes argentinos, el cual mostró correlaciones de kappa sobre 0,6 en base a preguntas directas y sencillas $^{10}$.

Tampoco se puede obviar el efecto que tiene la variabilidad interobservador entre los distin- 
tos médicos participantes, factor que también puede afectar en los resultados del trabajo.

En resumen, podemos concluir que la autoevaluación del desarrollo puberal mediante la escala de Tanner es una herramienta poco confiable para su uso en la población escolar de ambos sexos y no debería utizarse para establecer decisiones clínicas. Sería de interés evaluar otros instrumentos como una escala o cuestionario diferente, que sea más fácil de realizar y de comprender por los niños y adolescentes a los que se va a aplicar.

\section{Referencias}

1.- Marshall WA, Tanner JM: Variations in Pattern of Pubertal Changes in Girls. Arch Dis Child 1969 44: 291-303.

2.- Marshall WA, Tanner JM: Variations in the Pattern of Pubertal Changes in Boys. Arch Dis Child 1970 45: 1323.

3.- Desmangles JC, Lappe JM, Lipaczewski G, Haynatzki G: Accuracy of pubertal Tanner staging self-reporting. J Pediatr Endocrinol Metab 2006; 19 (3): 213-21.

4.- Azevedo JCV, Brasil LMP, Macedo TBMA, Pedrosa
LFC, Arrais RF: Comparison between objective assessment and self-assessment of sexual maturation in children and adolescents. J Pediatr (Rio J) 2009; 85 (2): 135-42.

5.- Raman A, Lustig R, Fitch M, Fleming S: Accuracy of Self-assessed Tanner Staging Against Hormonal Assessment of Sexual Maturation in Overweight AfricanAmerican Children. J Pediatr Endocrinol Metab2009, 22 (7), 609-22.

6.- Lee K, Valeria B, Kochman C, Lenders C: Selfassessment of Height, Weight, and Sexual Maturation: Validity in Overweight Children and Adolescents. J Adolesc Health 2006 (39) 346-52.

7.- Bonat S, Pathomvanich A, Keil M, Field A, Yanovski $J$ : Self-Assessment of Pubertal Stage in Overweight Children. Pediatrics 2002; 110: 743-7.

8.- Kuczmarski RJ, Ogden CL, Grummer-Strawn LM, et al: CDC growth charts: United States. Adv Data. 2000; 314: 1-27.

9.- Cerda J, Vilarroel L: Evaluación de la concordancia inter-observador en investigación pediátrica: Coeficiente de Kappa. Rev Chil Pediatr 2008; 79 (1): 54-8.

10.- Lejarragaa H, Enrique Bernerb E, del Pinoa M, Medinab V, Cameron N: Método no invasivo para la evaluación del desarrollo sexual en la adolescencia. Arch Argent Pediatr 2009; 107 (5): 423-9. 